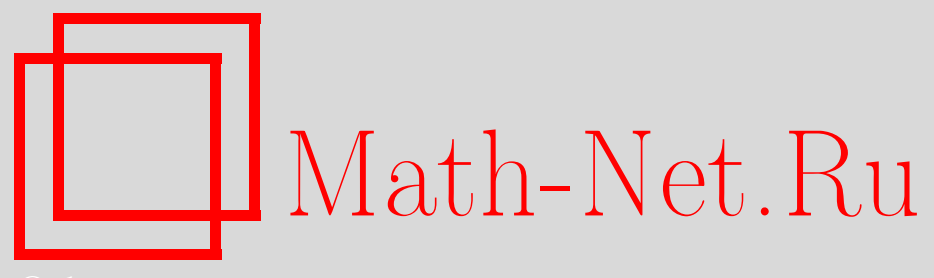

C.-Х. Ван, Г. Лв, Глобальная устойчивость фронтов бегущих волн для нелокального уравнения диффузии с запаздыванием, Изв. РАН. Сер. матем., 2014, том 78, выпуск $2,43-60$

DOI: https://doi.org/10.4213/im8062

Использование Общероссийского математического портала Math-Net.Ru подразумевает, что вы прочитали и согласны с пользовательским соглашением http://www . mathnet.ru/rus/agreement

Параметры загрузки:

IP : 54.198 .187 .58

26 апреля 2023 г., 12:53:18

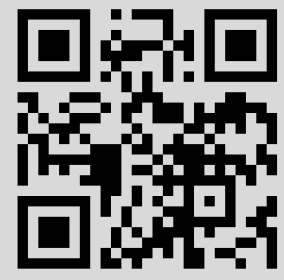




\author{
С.-Х. Ван, Г. Лв
}

\title{
Глобальная устойчивость фронтов бегущих волн для нелокального уравнения диффузии с запаздыванием
}

Изучается вопрос о глобальной устойчивости фронтов бегущих волн для нелокальных уравнений диффузии с запаздыванием. Показано, что фронты некритических бегущих волн глобально экспоненциально устойчивы при возмущениях из пространств $L^{\infty}$ с некоторым экспоненциальным весом. С помощью весовых энергетических оценок найдена скорость убывания по времени величины $\sup _{x \in \mathbb{R}}|u(x, t)-\varphi(x+c t)|$.

Библиография: 25 наименований.

Ключевые слова: устойчивость, нелокальные уравнения реакциидиффузии, запаздывание, фронт бегущей волны, весовая энергетическая оценка.

DOI: $10.4213 / \operatorname{im} 8062$

\section{§ 1. Введение}

Мы изучаем вопрос о глобальной устойчивости фронтов бегущих волн для следующего нелокального уравнения реакции-диффузии с запаздыванием:

$$
u_{t}(x, t)=(J * u)(x, t)-u(x, t)+f(u(x, t), u(x, t-\tau)), \quad x \in \mathbb{R}, \quad t>0,
$$

при начальных условиях

$$
u(x, s)=u_{0}(x, s), \quad x \in \mathbb{R}, \quad s \in[-\tau, 0],
$$

где $\tau \geqslant 0$ - неотрицательная константа, $J \in C^{1}(\mathbb{R})$ - неотрицательная четная функция такая, что

$$
\int_{\mathbb{R}} J(y) \mathrm{d} y=1, \quad \int_{\mathbb{R}} J(y) e^{\lambda y} \mathrm{~d} y<\infty
$$

для всех $\lambda \in \mathbb{R}$, и где используется обозначение

$$
J * u=\int_{\mathbb{R}} J(x-y) u(y, t) \mathrm{d} y .
$$

Работа первого автора выполнена при частичной поддержке Национального научного фонда Китайской Народной Республики (грант NSFC 11226168), работа второго автора при частичной поддержке Национального научного фонда Китайской Народной Республики (гранты 11171064 и 11226168) и Фонда естественных наук провинции Цзяньсу (грант BK2011583). 
Хорошо известно, что при $\tau=0$ существует единственный (с точностью до сдвига) фронт бегущей волны для уравнения (1.1) (см. работы [1]-[3] и ссылки в них). Асимптотическая устойчивость фронта бегущей волны для уравнения (1.1) при $\tau=0$ была получена Ченом [3] методом просачивания. В [4], [5] установлена многомерная устойчивость фронта бегущей волны для уравнения (1.1) при $\tau=0$ с помощью спектрального анализа.

Недавно в работе [6] было рассмотрено уравнение (1.1) с квазимонотонной функцией $f$ и установлено существование фронта бегущей волны с помощью теоремы Шаудера о неподвижной точке и техники суб- и суперрешений. В [7] методами работы [8] изучалась устойчивость фронтов быстро движущихся волн уравнения (1.1), где $f(u, v)=-a u+b v(1-u)$, т. е. уравнения

$$
u_{t}-J * u+u+a u=b u(x, t-\tau)(1-u), \quad b>a \geqslant 0,
$$

в предположении существования положительной константы $\eta$ такой, что

$$
\int_{0}^{+\infty} J(y) e^{\eta y} \mathrm{~d} y<\frac{1}{2}+\frac{2}{3}(b-a) .
$$

В настоящей работе по-новому рассматривается устойчивость фронтов бегущих волн для уравнения (1.1), где $f(u, v)=-a u+b v(1-u)$, и мы не налагаем ограничения (1.4).

Устойчивость бегущих волн широко изучалась различными авторами. Д. Саттингер [9] показал с помощью подробного спектрального анализа, что фронты бегущих волн для систем реакции-диффузии без запаздывания устойчивы относительно возмущений из пространств $L^{\infty}$ с некоторым экспоненциальным весом. Х. Л. Смит и Х. К. Чао [10] рассматривали уравнение (1.1) с заменой $J * u-u$ на $\Delta u$. Они доказали теоремы существования и сравнения для решений квазимонотонных биустойчивых уравнений реакции-диффузии с дискретным запаздыванием и получили устойчивость фронтов бегущих волн с помощью элементарных методов сравнения суб- и суперрешений и методов просачивания, развитых в [3] (об этой технике см. также [11]-[13]).

Совсем недавно в работе [14] было рассмотрено следующее уравнение реакции-диффузии с запаздыванием и нелокальной нелинейностью:

$$
u_{t}-D u_{x x}+d(u)=\varepsilon \int_{-\infty}^{+\infty} f_{\alpha}(y) b(u(x-y, t-\tau)) \mathrm{d} y, \quad x \in \mathbb{R}, \quad t>0,
$$

при начальных условиях $(1.2)$, где $D, \varepsilon>0$ - константы, $f_{\alpha}(y)$ - ядро теплопроводности, а нелинейные функции $d(u)$ и $b(u)$ - уровни смертности и рождаемости во взрослой популяции. В указанной работе установлено, что фронты всех некритических бегущих волн глобально экспоненциально устойчивы, а фронты критических волн глобально алгебраически устойчивы при условии, что начальное возмущение фронта экспоненциально убывает на минус бесконечности независимо от величины запаздывания по времени. Этот результат дополняет предыдущие достижения в данной области [8], [15]-[18]. Нам не известны какие-либо результаты об устойчивости фронтов низших бегущих волн для уравнения (1.3). 
Между тем, уравнения вида $u_{t}=J * u-u$ и их модификации в последнее время широко используются для моделирования диффузионных процессов, например, в биологии, динамике дислокаций и других областях (см. детали в [19]-[21]). Согласно [22] если считать $u(x, t)$ плотностью популяции в точке $x$ в момент времени $t$, а $J(x-y)$ - распределением вероятности скачка из положения $y$ в положение $x$, то $(J * u)(x, t)=\int_{\mathbb{R}} J(x-y) u(y, t) \mathrm{d} y$ представляет собой скорость, с которой особи прибывают в точку $x$ из других мест, а $-u(x, t)=-(J * u)(x, t)$ есть скорость, с которой они покидают точку $x$, отправляясь в другие места. В отсутствие внешних или внутренних источников отсюда вытекает, что плотность $u$ удовлетворяет уравнению $u_{t}=J * u-u$. С другой стороны, Дж. Д. Мюррей [23] отмечает, что общие уравнения реакции-диффузии

$$
u_{t}=d u_{x x}+f(u), \quad x \in \mathbb{R}, \quad t>0,
$$

вполне применимы только к редким системам и моделям популяций. В реальности же обычно присутствует запаздывание во времени, что делает уравнение (1.1) более адекватной моделью.

Вдохновляясь работой [14], мы изучим в настоящей статье вопрос о глобальной устойчивости фронтов бегущих волн для уравнения (1.1). Отметим значительную разницу между уравнениями (1.3) и (1.5), делающую доказательство устойчивости фронтов бегущих волн для (1.3) нетривиальным. Во-первых, оператор Лапласа $\Delta u$ гораздо проще, чем оператор диффузии $J * u-u$. Во-вторых, М. Мей и соавторы [14] сначала доказывают весовые $L^{1}$-энергетические оценки для решений, пользуясь выпуклостью нелинейных функций рождаемости и смертности, а затем получают требуемую $L^{2}$-энергетическую оценку. Мы же из-за наличия оператора диффузии $J * u-u$ не можем получить $L^{2}$-энергетическую оценку методами работы [14] и устанавливаем для этого ряд новых оценок. Сначала $L^{2}$-энергетическая оценка будет получена на некотором подынтервале в $\mathbb{R}$, а затем с помощью свойств бегущих волн распространена на другие подынтервалы в $\mathbb{R}$.

В процессе написания работы выяснилось, что Р. Хуан с соавторами [24] рассмотрели задачу

$$
\begin{aligned}
u_{t}-J * u+u+d(u) & =\int_{-\mathbb{R}^{n}} f_{\alpha}(y) b(u(x-y, t-\tau)) \mathrm{d} y, \quad x \in \mathbb{R}^{n}, \quad t>0, \\
u(x, s) & =u_{0}(x, s), \quad x \in \mathbb{R}^{n}, \quad s \in[-\tau, 0],
\end{aligned}
$$

где $f_{\alpha}$ определяется, как в (1.5), и показали устойчивость плоских волн для этой задачи с помощью преобразования Фурье и принципа сравнения при условии, что

$$
\widehat{J}(\xi)=1-\mathcal{K}|\xi|^{\alpha}+O\left(|\xi|^{\alpha}\right), \quad \xi \rightarrow 0, \quad \alpha \in(0,2),
$$

где $\mathcal{K}>0$ и $\widehat{J}$ - преобразование Фурье функции $J$. Мы увидим, что при выполнении условия (1.7) можно получить устойчивость фронтов некритических и критических бегущих волн уравнения (1.1). 
Всюду далее через $C>0$ обозначена общая константа, а $C_{i}, i=1,2, \ldots,-$ конкретные константы. Через $I$ обозначен интервал (обычно $I=\mathbb{R}$ ), через $L^{2}(I)$ - пространство квадратично интегрируемых функций на $I$, а через $H^{k}(I), k \geqslant 0,-$ соболевское пространство тех $L^{2}$-функций $f(x)$ на $I$, производные $\frac{\mathrm{d}^{i}}{\mathrm{~d} x^{i}} f, i=1, \ldots, k$, которых также лежат в $L^{2}(I)$. Весовое $L^{2}$-пространство с весом $w(x)>0$ обозначается через $L_{w}^{2}(I)$ и снабжается нормой

$$
\|f\|_{L_{w}^{2}(I)}=\left(\int_{I} w(x)|f(x)|^{2} \mathrm{~d} x\right)^{1 / 2},
$$

а весовое соболевское пространство $H_{w}^{k}(I)$ снабжается нормой

$$
\|f\|_{H_{w}^{k}(I)}=\left(\sum_{i=0}^{k} \int_{I} w(x)\left|\frac{\mathrm{d}^{i} f(x)}{\mathrm{d} x^{i}}\right|^{2} \mathrm{~d} x\right)^{1 / 2} .
$$

Для заданного числа $T>0$ и банахова пространства $B$ обозначим через $C^{0}([0, T] ; B)$ пространство всех непрерывных $B$-значных функций на $[0, T]$, а через $L^{2}([0, T] ; B)$ - пространство всех $B$-значных $L^{2}$-функций на $[0, T]$. Аналогично определяется пространство $B$-значных $L^{2}$-функций на $[0, \infty)$.

Структура работы следующая. В $§ 2$ определяются фронты бегущих волн и формулируется теорема об устойчивости. В 33 приведено доказательство этой теоремы. Статья заканчивается кратким обсуждением полученных результатов.

\section{§ 2. Предварительные сведения и формулировка основной теоремы}

Сначала мы напомним некоторые известные результаты, затем определим весовые функции и, наконец, сформулируем основную теорему. Поскольку $a<b$, легко видеть, что уравнение (1.3) имеет два неотрицательных постоянных состояния равновесия: $u_{-}=0$ и $u_{+}=1-a / b$.

Фронтом бегущей волны уравнения (1.3), связанной с состояниями равновесия $u_{ \pm}$, называется решение $u$ вида $u(x, t)=\varphi(x+c t)$ с условиями $\varphi^{\prime}(\xi) \geqslant 0$ и

$$
\begin{gathered}
c \varphi^{\prime}(\xi)=(J * \varphi)(\xi)-\varphi(\xi)-a \varphi(\xi)+b \varphi(\xi-c \tau)(1-\varphi(\xi)), \\
\varphi( \pm \infty)=u_{ \pm},
\end{gathered}
$$

где $\xi=x+c t$ и штрих означает $\frac{\mathrm{d}}{\mathrm{d} \xi}$. В частном случае $a=0$ существование фронта бегущей волны было доказано в [6]. В работе [7] установлено, что задача (2.1) обладает по крайней мере одним решением. Полагая

$$
\Delta_{c}(\lambda)=\int_{\mathbb{R}} J(y) e^{\lambda y} \mathrm{~d} y-1-c \lambda-a+b e^{-\lambda c \tau},
$$

имеем следующее

ПредлОЖениЕ 2.1. Если $\Delta_{c}(\lambda)$ определено формулой (2.2), то существуют минимальная скорость волны $c^{*}>0$ (называемая также критической 
скоростью волны) и число $\lambda^{*}$, удовлетворяющие условиям

$$
\Delta_{c^{*}}\left(\lambda^{*}\right)=0, \quad \frac{\partial}{\partial \lambda} \Delta_{c^{*}}\left(\lambda^{*}\right)=0
$$

и такие, что для каждого $c \geqslant c^{*}$ уравнение (1.3) имеет решение вида $\varphi(x+c t)$ с условиями $\varphi( \pm \infty)=u_{ \pm}$. При этом для $c>c^{*}$ выполнено

$$
\lim _{\xi \rightarrow-\infty} \varphi(\xi) e^{-\lambda_{1}(c) \xi}=1, \quad \lim _{\xi \rightarrow-\infty} \varphi^{\prime}(\xi) e^{-\lambda_{1}(c) \xi}=\lambda_{1}(c),
$$

где $\lambda_{1}(c)$ - наименьший корень уравнения $\Delta_{c}(\lambda)=0$.

ДокАзАтеЛьство. Легко видеть, что существуют константы $c^{*}>0$ и $\lambda^{*}$, для которых выполнены условия (2.3). При этом уравнение $\Delta_{c}(\lambda)=0$ имеет два различных положительных корня для $c>c^{*}$ и не имеет вещественных корней для $c<c^{*}$. Точнее, найдутся две константы $0<\lambda_{1}(c)<\lambda_{2}(c)$ такие, что $\Delta_{c}(\lambda)>0$ при $0<\lambda<\lambda_{1}(c), \Delta_{c}(\lambda)=0$ при $\lambda=\lambda_{1}(c), \lambda_{2}(c), \Delta_{c}(\lambda)<0$ при $\lambda_{1}(c)<\lambda<\lambda_{2}(c), \Delta_{c}(\lambda)>0$ при $\lambda>\lambda_{2}(c)$.

Докажем существование фронта бегущей волны методом суб- и суперрешений. Положим

$$
\bar{\varphi}(\xi)=\min \left\{1-\frac{a}{b}, e^{\lambda_{1}(c) \xi}\right\}, \quad \underline{\varphi}(\xi)=\max \left\{0, e^{\lambda_{1}(t) \xi}-q e^{\eta \lambda_{1}(c) \xi}\right\},
$$

где $q>1$ достаточно велико и

$$
\eta \in\left(1, \min \left\{2, \frac{\lambda_{2}(c)}{\lambda_{1}(c)}\right\}\right) .
$$

Легко проверяется, что $\bar{\varphi}(\xi)$ и $\underline{\varphi}(\xi)$ образуют пару из суперрешения и субрешения уравнения (2.1). Мы оставляем детали читателю (см. также [6, леммы 4.4, 4.5]). Теперь из [6, теорема 3.2] можно вывести предложение.

Заметим, что

$$
\Delta_{c}(\lambda)<0 \quad \forall c>c^{*}, \quad \lambda \in\left(\lambda_{1}(c), \lambda_{2}(c)\right)
$$

Для таких $c>c^{*}$ и $\lambda \in\left(\lambda_{1}(c), \lambda^{*}\right)$ определим две весовые функции формулами

$$
w_{1}(\xi)=e^{-\lambda \xi}, \quad w_{2}(\xi)=e^{-2 \lambda \xi} .
$$

Мы выбираем $\lambda \in\left(\lambda_{1}(c), \lambda^{*}\right)$ для того, чтобы гарантировать более быстрое убывание $\left|u_{0}(x, s)-\varphi(x+c s)\right|$ по сравнению с $\left|\varphi(x+c s)-\varphi\left(x+c s+x_{0}\right)\right|$, где $x_{0}-$ произвольный сдвиг.

ТЕОРема 2.1. Для любого фронта бегущей волны $\varphi(x+c t)$ уравнения (1.3) со скоростью $c>c^{*}$ и условиями $\varphi( \pm \infty)=u_{ \pm}$, если начальные данные удовлетворяют

$$
u_{-} \leqslant u_{0}(x, s) \leqslant u_{+}, \quad(x, s) \in \mathbb{R} \times[-\tau, 0],
$$


а начальное возмущение $u_{0}(x, s)-\varphi(x+c s)$ принадлежит $C\left([-\tau, 0] ; L_{w}^{1}(\mathbb{R}) \cap\right.$ $\left.H_{w}^{1}(\mathbb{R})\right)$, решение задачи $(1.1),(1.2)$ существует, является единственным и удовлетворяет условиям

$$
\begin{gathered}
u_{-} \leqslant u(x, t) \leqslant u_{+}, \quad(x, t) \in \mathbb{R} \times \mathbb{R}_{+}, \\
u(x, t)-\varphi(x+c t) \in C\left([0,+\infty) ; L_{w}^{1}(\mathbb{R}) \cap H_{w}^{1}(\mathbb{R})\right)
\end{gathered}
$$

для веса $w(x)=\max \left\{w_{1}(x), w_{2}(x)\right\}$, где функиии $w_{1}(x), w_{2}(x)$ заданы равенствами (2.4). При этом решение $u(x, t)$ сходится $\kappa$ фронту бегущей волнъ $\varphi(x+c t)$ экспоненииально по времени:

$$
\sup _{x \in \mathbb{R}}|u(x, t)-\varphi(x+c t)| \leqslant C e^{-\mu t}
$$

для некоторых положительных констант $C u \mu$.

\section{§ 3. Доказательство теоремы 2.1}

Сначала мы установим принцип сравнения для задачи (1.1), (1.2), а затем докажем теорему 2.1 весовым энергетическим методом, аналогично работам [8], [14], [18].

Лемма 3.1. Пусть $T>0$ и $Q_{T}=\mathbb{R} \times(0, T]$. Допустим, что неотрицательная функиия $c(x, t)$ ограничена при $(x, t) \in Q_{T}$. Если функиия и удовлетворяет неравенствам

$$
\begin{gathered}
u_{t}-J * u+u+c(x, t) u \geqslant 0(\leqslant 0), \quad(x, t) \in Q_{T}, \\
u(x, 0) \geqslant 0(\leqslant 0), \quad x \in \mathbb{R},
\end{gathered}
$$

mo $u(x, t) \geqslant 0(\leqslant 0)$ nрu $(x, t) \in Q_{T}$.

Мы не приводим простое доказательство этой леммы. Заметим, что уравнение $u_{t}=J * u-u$ имеет много общих свойств с уравнением теплопроводности $u_{t}=\Delta u$. В частности, все его ограниченные стационарные решения постоянны, и справедлив принцип максимума (см. детали в [25]). Поэтому, пользуясь леммой 3.1 и рассуждая, как при доказательстве теоремы 2.1 и леммы 2.1 из работы [18], можно установить следующие леммы, где предполагается, что $f\left(u_{ \pm}, u_{ \pm}\right)=0, f(u, v)>0$ для $(u, v) \in\left(u_{-}, u_{+}\right)^{2}, \partial_{v} f(u, v) \geqslant 0$ и $\partial_{u} f(u, v)$ ограничена при $(u, v) \in\left[u_{-}, u_{+}\right]^{2}$.

Лемма 3.2. Пусть $u_{-}=0 \leqslant u_{0}(x, s) \leqslant u_{+}$при $(x, s) \in \mathbb{R} \times[-\tau, 0]$. Тогда решение $и(x, t)$ задачи Коши (1.1), (1.2) удовлетворяет неравенствам $u_{-} \leqslant$ $u(x, t) \leqslant u_{+} n p u(x, t) \in \mathbb{R} \times \mathbb{R}_{+}$.

ЛЕмма 3.3 (принцип сравнения). Пусть $u^{-}(x, t) u u^{+}(x, t)$ - решения уравнения (1.1) с начальными условиями $u_{0}^{-}(x, s)$ и $u_{0}^{+}(x, s)$ соответственно. Если

$$
u_{-} \leqslant u_{0}^{-}(x, s) \leqslant u_{0}^{+}(x, s) \leqslant u_{+}, \quad(x, s) \in \mathbb{R} \times[-\tau, 0],
$$

mo

$$
u_{-} \leqslant u^{-}(x, t) \leqslant u^{+}(x, t) \leqslant u_{+}, \quad(x, t) \in \mathbb{R} \times \mathbb{R}_{+} .
$$

Очевидно, леммы 3.2, 3.3 справедливы и для уравнения (1.3). 
Следуя идее работ [8], [14], [18], применим принцип сравнения и весовой энергетический метод для доказательства теоремы 2.1. Пусть начальное условие $u_{0}(x, s)$ таково, что

$$
u_{-}=0 \leqslant u_{0}(x, s) \leqslant u_{+}, \quad(x, s) \in \mathbb{R} \times[-\tau, 0] .
$$

Положим

$$
\begin{array}{ll}
u_{0}^{-}(x, s)=\min \left\{u_{0}(x, s), \varphi(x+c s)\right\}, & (x, s) \in \mathbb{R} \times[-\tau, 0], \\
u_{0}^{+}(x, s)=\max \left\{u_{0}(x, s), \varphi(x+c s)\right\}, & (x, s) \in \mathbb{R} \times[-\tau, 0] .
\end{array}
$$

Ясно, что

$$
\begin{array}{ll}
u_{-} \leqslant u_{0}^{-}(x, s) \leqslant u_{0}(x, s) \leqslant u_{0}^{+}(x, s) \leqslant u_{+}, & (x, s) \in \mathbb{R} \times[-\tau, 0], \\
u_{-} \leqslant u_{0}^{-}(x, s) \leqslant \varphi(x+c s) \leqslant u_{0}^{+}(x, s) \leqslant u_{+}, & (x, s) \in \mathbb{R} \times[-\tau, 0] .
\end{array}
$$

Обозначим через $u^{-}(x, t)$ и $u^{+}(x, t)$ решения задачи $(1.3),(1.2)$ с начальными условиями $u_{0}^{-}(x, s)$ и $u_{0}^{+}(x, s)$ соответственно. Согласно принципу сравнения имеем

$$
\begin{array}{ll}
u_{-} \leqslant u^{-}(x, t) \leqslant u(x, t) \leqslant u^{+}(x, t) \leqslant u_{+}, & (x, s) \in \mathbb{R} \times \mathbb{R}_{+}, \\
u_{-} \leqslant u^{-}(x, t) \leqslant \varphi(x+c t) \leqslant u^{+}(x, t) \leqslant u_{+}, & (x, s) \in \mathbb{R} \times \mathbb{R}_{+} .
\end{array}
$$

ДокАЗАТЕЛЬСтво теоремы 2.1. Докажем теорему в три шага.

Шаг 1. Докажем сходимость $u^{+}(x, t) \mathrm{k} \varphi(x+c t)$. Положим $\xi=x+c t$ и определим

$$
v(\xi, t)=u^{+}(x, t)-\varphi(x+c t), \quad v_{0}(\xi, s)=u_{0}^{+}(x, s)-\varphi(x+c s) .
$$

Из (3.2) и (3.4) вытекает, что $v(\xi, t) \geqslant 0, v_{0}(\xi, s) \geqslant 0$. Кроме того, $v(\xi, t)$ удовлетворяет равенствам

$$
\begin{gathered}
v_{t}+c v_{\xi}-J * v+v+(a+b \varphi(\xi-c \tau)) v \\
=b(1-v-\varphi) v(\xi-c \tau, t-\tau), \quad(\xi, t) \in \mathbb{R} \times \mathbb{R}_{+}, \\
v(\xi, s)=v_{0}(\xi, s), \quad(\xi, s) \in \mathbb{R} \times[-\tau, 0] .
\end{gathered}
$$

ЛЕмма 3.4. Имеем

$$
\|v(t)\|_{L_{w_{1}}^{1}(\mathbb{R})} \leqslant C e^{-\mu_{1} t}, \quad c>c^{*},
$$

где $\mu_{1}>0$ таково, что

$$
J * e^{\lambda \cdot}-1-c \lambda+\mu_{1}-a+b e^{\mu_{1} \tau} e^{-\lambda c \tau}<0, \quad \lambda \in\left(\lambda_{1}(c), \lambda^{*}\right) .
$$

ДокАЗАтЕльство. С технической точки зрения для доказательства энергетической оценки (3.6) необходима достаточная регулярность решений задачи (3.5). Она обычно устанавливается с помощью сглаживания (см. детали 
в [14], [18]). Начиная отсюда будем считать, что решение $v(\xi, t)$ достаточно регулярно.

Установим теперь (3.6) для всех $t>0$. Умножая первое уравнение (3.5) на $w_{1}(\xi) e^{\mu_{1} t}$, где $\mu_{1}>0$ определяется, как в $(3.7)$, имеем

$$
\begin{aligned}
&\left(e^{\mu_{1} t} w_{1} v\right)_{t}+e^{\mu_{1} t}\left(c w_{1} v\right)_{\xi}- e^{\mu_{1} t} w_{1}(\xi) \int_{\mathbb{R}} J(y) v(\xi-y, t) \mathrm{d} y \\
&+\left[-c w_{1}^{\prime}+w_{1}-\mu_{1} w_{1}+w_{1}(a+b \varphi(\xi-c \tau))\right] e^{\mu_{1} t} w_{1} v \\
&=b v(\xi-c \tau, t-\tau)(1-v-\varphi) w_{1} e^{\mu_{1} t}
\end{aligned}
$$

Интегрируя последнее равенство по $\xi$ и $t$ на $\mathbb{R} \times[0, t]$, получаем

$$
\begin{aligned}
& e^{\mu_{1} t} \int_{\mathbb{R}} w_{1}(\xi) v(\xi, t) \mathrm{d} \xi+\int_{0}^{t} \int_{\mathbb{R}} e^{\mu_{1} s} w_{1}(\xi) \int_{\mathbb{R}} J(y) v(\xi-y, s) \mathrm{d} y \mathrm{~d} \xi \mathrm{d} s \\
& \quad+\int_{0}^{t} \int_{\mathbb{R}}\left[-c w_{1}^{\prime}(\xi)+w_{1}(\xi)-\mu_{1} w_{1}(\xi)+w_{1}(\xi)(a+b \varphi(\xi-c \tau))\right] e^{\mu_{1} s} v(\xi, s) \mathrm{d} \xi \mathrm{d} s \\
& =\left\|v_{0}(0)\right\|_{L_{w_{1}}^{1}}+b \int_{0}^{t} \int_{\mathbb{R}} v(\xi-c \tau, s-\tau)(1-v(\xi, s)-\varphi(\xi)) w_{1}(\xi) e^{\mu_{1} s} \mathrm{~d} \xi \mathrm{d} s . \quad(3.8)
\end{aligned}
$$

Второе слагаемое в левой части (3.8) имеет вид

$$
\begin{aligned}
\int_{0}^{t} \int_{\mathbb{R}} e^{\mu_{1} s} w_{1}(\xi) \int_{\mathbb{R}} J(y) & v(\xi-y, s) \mathrm{d} y \mathrm{~d} \xi \mathrm{d} s \\
= & \int_{0}^{t} \int_{\mathbb{R}} e^{\mu_{1} s} w_{1}(\xi) v(\xi, s) \int_{\mathbb{R}} J(y) \frac{w_{1}(\xi+y)}{w_{1}(\xi)} \mathrm{d} y \mathrm{~d} \xi \mathrm{d} s .
\end{aligned}
$$

Для правой части равенства (3.8) имеем

$$
\begin{gathered}
b \int_{0}^{t} \int_{\mathbb{R}} v(\xi-c \tau, s-\tau)(1-v(\xi, s)-\varphi(\xi)) w_{1}(\xi) e^{\mu_{1} s} \mathrm{~d} \xi \mathrm{d} s \\
=b e^{\mu_{1} \tau} \int_{-\tau}^{t-\tau} \int_{\mathbb{R}}(1-v(\xi+c \tau, s+\tau)-\varphi(\xi+c \tau)) v(\xi, s) w_{1}(\xi+c \tau) e^{\mu_{1} s} \mathrm{~d} \xi \mathrm{d} s \\
\leqslant b e^{\mu_{1} \tau} \int_{-\tau}^{0} \int_{\mathbb{R}}(1-\varphi(\xi+c \tau)) v_{0}(\xi, s) w_{1}(\xi+c \tau) e^{\mu_{1} s} \mathrm{~d} \xi \mathrm{d} s \\
+b e^{\mu_{1} \tau} \int_{0}^{t} \int_{\mathbb{R}}(1-\varphi(\xi+c \tau)) v(\xi, s) w_{1}(\xi+c \tau) e^{\mu_{1} s} \mathrm{~d} \xi \mathrm{d} s .
\end{gathered}
$$

Подставляя (3.9), (3.10) в (3.8), имеем

$$
\begin{aligned}
& e^{\mu_{1} t} \int_{\mathbb{R}} w_{1}(\xi) v(\xi, t) \mathrm{d} \xi+\int_{0}^{t} \int_{\mathbb{R}} A_{w_{1}}(\xi) w_{1}(\xi) e^{\mu_{1} s} v(\xi, s) \mathrm{d} \xi \mathrm{d} s \\
& \leqslant\left\|v_{0}(0)\right\|_{L_{w_{1}}^{1}}+b e^{\mu_{1} \tau} \int_{-\tau}^{0} \int_{\mathbb{R}}(1-\varphi(\xi+c \tau)) v_{0}(\xi, s) w_{1}(\xi+c \tau) e^{\mu_{1} s} \mathrm{~d} \xi \mathrm{d} s
\end{aligned}
$$

где

$$
\begin{array}{rl}
A_{w_{1}}(\xi)=-c \frac{w_{1}^{\prime}(\xi)}{w_{1}(\xi)}-\int_{\mathbb{R}} & J(y) \frac{w_{1}(\xi+y)}{w_{1}(\xi)} \mathrm{d} y+1-\mu_{1} \\
& \quad+(a+b \varphi(\xi-c \tau))-b e^{\mu_{1} \tau}(1-\varphi(\xi+c \tau)) \frac{w_{1}(\xi+c \tau)}{w_{1}(\xi)} .
\end{array}
$$


Замечая, что $w_{1}(\xi)=e^{-\lambda \xi}$, и пользуясь $(3.7)$, получаем

$$
\begin{aligned}
A_{w_{1}}(\xi) & =c \lambda-J * e^{\lambda \cdot}+1-\mu_{1}+a+b \varphi(\xi-c \tau)-b e^{\mu_{1} \tau}(1-\varphi(\xi+c \tau)) e^{-\lambda c \tau} \\
& \geqslant c \lambda-J * e^{\lambda \cdot}+1-\mu_{1}+a-b e^{\mu_{1} \tau} e^{-\lambda c \tau} \geqslant 0 .
\end{aligned}
$$

Подставляя это неравенство в (3.11), получаем требуемую оценку (3.6). Лемма доказана.

ЛЕмма 3.5. Имеем

$$
\left\|v_{\xi}(t)\right\|_{L^{2}(I)} \leqslant C, \quad c>c^{*}
$$

где $I=(-\infty, 0]$.

ДокАзАтельство. Покажем сначала, что $\|v(t)\|_{L_{w_{2}}^{2}} \leqslant C$, где вес $w_{2}(\xi)$ определяется равенством (2.4). Умножая (3.5) на $e^{2 \mu_{2} t} w_{2}(\xi) v(\xi, t)$, где $\mu_{2}>0$ будет выбрано далее, в лемме 3.7, мы имеем

$$
\begin{aligned}
&\left(\frac{1}{2} e^{2 \mu_{2} t} w_{2} v^{2}\right)_{t}+\left(\frac{c}{2} e^{2 \mu_{2} t} w_{2} v^{2}\right)_{\xi}-e^{2 \mu_{2} t} w_{2}(\xi) v(\xi, t) \int_{\mathbb{R}} J(y) v(\xi-y, t) \mathrm{d} y \\
&+\left(-\frac{c}{2} \frac{w_{2}^{\prime}}{w_{2}}-\mu_{2}+1+a+b \varphi(\xi-c \tau)\right) w_{2} v^{2} e^{2 \mu_{2} t} \\
&=b(1-v-\varphi) w_{2} v(\xi, t) v(\xi-c \tau, t-\tau) e^{2 \mu_{2} t}
\end{aligned}
$$

Интегрируя (3.13) по $(\xi, t)$ на $\mathbb{R} \times[0, t]$, получаем

$$
\begin{aligned}
& e^{2 \mu_{2} t}\|v(t)\|_{L_{w_{2}}^{2}}^{2}-2 \int_{0}^{t} \int_{\mathbb{R}} e^{2 \mu_{2} s} w_{2}(\xi) v(\xi, s) \int_{\mathbb{R}} J(y) v(\xi-y, s) \mathrm{d} y \mathrm{~d} \xi \mathrm{d} s \\
& \quad+\int_{0}^{t} \int_{\mathbb{R}} e^{2 \mu_{2} s}\left(-c \frac{w^{\prime}(\xi)}{w(\xi)}-2 \mu_{2}+2-2(a+b \varphi(\xi-c \tau))\right) w_{2}(\xi) v^{2}(\xi, s) \mathrm{d} \xi \mathrm{d} s \\
& \leqslant\left\|v_{0}(0)\right\|_{L_{w_{2}}^{2}}^{2}+2 b \int_{0}^{t} \int_{\mathbb{R}}(1-\varphi(\xi)) w_{2}(\xi) v(\xi, s) v(\xi-c \tau, s-\tau) e^{2 \mu_{2} s} \mathrm{~d} \xi \mathrm{d} s .
\end{aligned}
$$

Пользуясь неравенством Коши-Шварца $2 x y \leqslant \eta x^{2}+\frac{1}{\eta} y^{2}, \eta>0$, получим следующие оценки:

$$
\begin{aligned}
2 \int_{0}^{t} \int_{\mathbb{R}} e^{2 \mu_{2} s} w_{2}(\xi) v(\xi, s) \int_{\mathbb{R}} J(y) v(\xi-y, s) \mathrm{d} y \mathrm{~d} \xi \mathrm{d} s & \\
\leqslant & \int_{0}^{t} \int_{\mathbb{R}} e^{2 \mu_{2} s} w_{2}(\xi) v^{2}(\xi, s) \int_{\mathbb{R}} J(y) \eta(y) \mathrm{d} y \mathrm{~d} \xi \mathrm{d} s \\
& \quad+\int_{0}^{t} \int_{\mathbb{R}} e^{2 \mu_{2} s} w_{2}(\xi) \int_{\mathbb{R}} J(y) \frac{1}{\eta(y)} v^{2}(\xi-y, s) \mathrm{d} y \mathrm{~d} \xi \mathrm{d} s \\
= & \int_{0}^{t} \int_{\mathbb{R}} e^{2 \mu_{2} s} w_{2}(\xi) v^{2}(\xi, s) \int_{\mathbb{R}} J(y) \eta(y) \mathrm{d} y \mathrm{~d} \xi \mathrm{d} s \\
& +\int_{0}^{t} \int_{\mathbb{R}} e^{2 \mu s} w_{2}(\xi) v^{2}(\xi, s) \int_{\mathbb{R}} J(y) \frac{w_{2}(\xi+y)}{\eta(y) w_{2}(\xi)} \mathrm{d} y \mathrm{~d} \xi \mathrm{d} s,
\end{aligned}
$$




$$
\begin{aligned}
2 b \int_{0}^{t} \int_{\mathbb{R}}(1-\varphi(\xi)) w_{2}(\xi) v(\xi, s) v(\xi-c \tau, s-\tau) e^{2 \mu_{2} s} \mathrm{~d} \xi \mathrm{d} s \\
\leqslant b \int_{0}^{t} \int_{\mathbb{R}} e^{2 \mu_{2} s}(1-\varphi(\xi)) w(\xi)\left(\varrho v^{2}(\xi, s)+\frac{1}{\varrho} v^{2}(\xi-c \tau, s-\tau)\right) \mathrm{d} \xi \mathrm{d} s \\
=b \varrho \int_{0}^{t} \int_{\mathbb{R}} e^{2 \mu_{2} s}(1-\varphi(\xi)) w_{2}(\xi) v^{2}(\xi, s) \mathrm{d} \xi \mathrm{d} s \\
\quad \leqslant \quad \int_{0}^{t} \int_{\mathbb{R}} e^{2 \mu_{2} s}(1-\varphi(\xi)) w_{2}(\xi) v^{2}(\xi, s) \mathrm{d} \xi \mathrm{d} s \\
+\frac{b}{\varrho} e^{2 \mu_{2} \tau} \int_{0}^{t} \int_{\mathbb{R}} e^{2 \mu_{2} s}(1-\varphi(\xi+c \tau)) w_{2}(\xi+c \tau) v^{2}(\xi, s) \mathrm{d} \xi \mathrm{d} s \\
+\frac{b}{\varrho} e^{2 \mu_{2} \tau} \int_{-\tau}^{0} \int_{\mathbb{R}} e^{2 \mu_{2} s}(1-\varphi(\xi+c \tau)) w_{2}(\xi+c \tau) v^{2}(\xi, s) \mathrm{d} \xi \mathrm{d} s
\end{aligned}
$$

Подставляя $(3.15)$ и $(3.16)$ в $(3.14)$ и пользуясь тем, что $\mu_{2} \in\left(0, \mu_{3}\right)$ (см. лемму 3.7$)$, имеем

$$
\begin{aligned}
& e^{2 \mu_{2} t}\|v(t)\|_{L_{w_{2}}^{2}}^{2}+\int_{0}^{t} \int_{\mathbb{R}} e^{2 \mu_{2} s} B_{\varrho, \mu_{2}, w_{2}}(\xi) w_{2}(\xi) v^{2}(\xi, s) \mathrm{d} \xi \mathrm{d} s \\
& \leqslant\left\|v_{0}(0)\right\|_{L_{w_{2}}^{2}}^{2}+\frac{1}{\varrho} e^{2 \mu \tau} \int_{-\tau}^{0} \int_{\mathbb{R}} e^{2 \mu_{2} s}(1-\varphi(\xi+c \tau)) w_{2}(\xi+c \tau) v_{0}^{2}(\xi, s) \mathrm{d} \xi \mathrm{d} s \\
& \leqslant C_{1}\left(\left\|v_{0}(0)\right\|_{L_{w_{2}}^{2}}^{2}+\int_{-\tau}^{0}\left\|v_{0}(s)\right\|_{L_{w_{2}}^{2}}^{2} \mathrm{~d} s\right),
\end{aligned}
$$

где

$$
\begin{gathered}
B_{\varrho, \mu_{2}, w_{2}}(\xi)=A_{\eta, \varrho, w_{2}}(\xi)-2 \mu_{2}-b\left(e^{2 \mu_{2} \tau}-1\right)(1-\varphi(\xi+c \tau)) \frac{w(\xi+c \tau)}{\varrho w(\xi)} \\
A_{\eta, \varrho, w_{2}}(\xi)=-c \frac{w_{2}^{\prime}(\xi)}{w_{2}(\xi)}+2-\int_{\mathbb{R}} J(y) \frac{w_{2}(\xi+y)}{\eta(y) w_{2}(\xi)} \mathrm{d} y-\int_{\mathbb{R}} J(y) \eta(y) \mathrm{d} y \\
-2 \partial_{1} f(\varphi(\xi), \varphi(\xi-c \tau))-b \varrho(1-\varphi(\xi))-\frac{b}{\varrho}(1-\varphi(\xi+c \tau)) \frac{w_{2}(\xi+c \tau)}{\varrho w_{2}(\xi)} .
\end{gathered}
$$

Чтобы получить основную оценку, надо установить, что $B_{\varrho, \mu_{2}, w_{2}}(\xi) \geqslant C>0$ для некоторой константы $C$. Для этого понадобится следующая ключевая лемма.

Лемма 3.6. Пусть $\eta(y)=e^{-\lambda y} u \varrho=e^{-c \tau \lambda}$. Тогда существует положительная константа $C_{2}$ такая, что

$$
A_{\eta, \varrho, w_{2}}(\xi) \geqslant C_{2}, \quad \xi \in \mathbb{R} .
$$


ДокАЗАТЕЛЬство. Из (2.4) вытекает, что $\frac{w_{2}(\xi+c \tau)}{w_{2}(\xi)}=e^{-2 \lambda c \tau}$ и $\frac{w(\xi+y)}{\eta(y) w(\xi)}=$ $e^{-\lambda y}$. Замечая, что $J(-y)=J(y)$, и пользуясь $(3.7)$, имеем

$$
\begin{aligned}
A_{\eta, \varrho, w_{2}}(\xi)=- & c \frac{w_{2}^{\prime}(\xi)}{w_{2}(\xi)}+2-\int_{\mathbb{R}} J(y) \frac{w_{2}(\xi+y)}{\eta(y) w_{2}(\xi)} \mathrm{d} y-\int_{\mathbb{R}} J(y) \eta(y) \mathrm{d} y \\
& +2(a+b \varphi(\xi-c \tau))-b \varrho(1-\varphi(\xi))-\frac{b}{\varrho}(1-\varphi(\xi+c \tau)) \frac{w_{2}(\xi+c \tau)}{\varrho w_{2}(\xi)} \\
= & 2 c \lambda-2 J * e^{\lambda \cdot}+2+2(a+b \varphi(\xi-c \tau)) \\
\geqslant & \quad-b e^{-\lambda c \tau}(1-\varphi(\xi))-b e^{-\lambda c \tau}(1-\varphi(\xi+c \tau)) \\
\geqslant & 2\left(c \lambda-J * e^{\lambda \cdot}+1-a-b e^{-\lambda c \tau}\right):=C_{2}>0 .
\end{aligned}
$$

Лемма доказана.

ЛЕмма 3.7. Пусть $\mu_{3}>0$ - единственный корень уравнения

$$
\frac{C_{2}}{2}-2 \mu_{3}-b\left(e^{2 \mu_{3} \tau}-1\right)=0 .
$$

Тогда $B_{\varrho, \mu_{2}, w_{2}}(\xi) \geqslant C_{3}:=C_{2} / 2>0$ на $\mathbb{R}$ при $0<\mu_{2} \leqslant \mu_{3}$.

ДокАЗАТЕЛЬСтво. Поскольку $\frac{w_{2}(\xi+c \tau)}{w_{2}(\xi)}=e^{-2 \lambda c \tau} \leqslant 1$, при $0<\mu_{2} \leqslant \mu_{3}$ имеем

$$
\begin{aligned}
B_{\varrho, \mu_{2}, w_{2}}(\xi) & =A_{\eta, \varrho, w_{2}}(\xi)-2 \mu_{2}-(1-\varphi(\xi+c \tau))\left(e^{2 \mu_{2} \tau}-1\right) \frac{w_{2}(\xi+c \tau)}{w_{2}(\xi)} \\
& \geqslant C_{2}-2 \mu_{2}-b\left(e^{2 \mu_{2} \tau}-1\right):=C_{3}>0 .
\end{aligned}
$$

Лемма доказана.

Подставляя результат леммы 3.7 в (3.17), имеем

$$
e^{2 \mu_{2} t}\|v(t)\|_{L_{w_{2}}^{2}}^{2}+C_{3} \int_{0}^{t} e^{2 \mu_{2} s}\|v(s)\|_{L_{w_{2}}^{2}}^{2} \mathrm{~d} s \leqslant C_{1}\left(\left\|v_{0}(0)\right\|_{L_{w}^{2}}^{2}+\int_{-\tau}^{0}\left\|v_{0}(s)\right\|_{L_{w}^{2}}^{2} \mathrm{~d} s\right) .
$$

Пренебрегая положительным слагаемым $\int_{0}^{t} e^{2 \mu_{2} s}\|v(s)\|_{L_{w}^{2}}^{2} \mathrm{~d} s$, получаем основную оценку

$$
e^{2 \mu_{2} t}\|v(t)\|_{L_{w_{2}}^{2}}^{2} \leqslant C_{1}\left(\left\|v_{0}(0)\right\|_{L_{w}^{2}}^{2}+\int_{-\tau}^{0}\left\|v_{0}(s)\right\|_{L_{w}^{2}}^{2} \mathrm{~d} s\right) .
$$

Теперь, дифференцируя (3.5) по $\xi$ и умножая полученное равенство на $e^{2 \mu_{2} t} w_{2}(\xi) v_{\xi}(\xi, t)$, где вес $w_{2}(\xi)$ задается формулой $(2.4)$, получаем

$$
\begin{aligned}
&\left(\frac{1}{2} e^{2 \mu_{2} t} w_{2} v_{\xi}^{2}\right)_{t}+\left(\frac{c}{2} e^{2 \mu_{2} t} w_{2} v_{\xi}^{2}\right)_{\xi}-e^{2 \mu_{2} t} w_{2}(\xi) v_{\xi}(\xi, t) \int_{\mathbb{R}} J(y) v_{\xi}(\xi-y, t) \mathrm{d} y \\
&+\left(-c \frac{w_{2}^{\prime}}{2 w_{2}}-\mu_{2}+1+a+b \varphi(\xi-c \tau)\right) w_{2} v_{\xi}^{2} e^{2 \mu_{2} t} \\
&=b(1-\varphi-v) w_{2} v_{\xi} v_{\xi}(\xi-c \tau, t-\tau) e^{2 \mu_{2} t}-b v(\xi-c \tau, t-\tau) v_{\xi}^{2} w_{2} e^{2 \mu_{2} t} \\
&-b \varphi_{\xi}(\xi-c \tau) v v_{\xi} w_{2} e^{2 \mu_{2} t}-b \varphi_{\xi} v_{\xi} w_{2} e^{2 \mu_{2} t} v(\xi-c \tau, t-\tau) .
\end{aligned}
$$


Интегрируя последнее равенство по $\mathbb{R} \times[0, t]$ и выполняя те же шаги, что и при выводе оценки (3.18), получаем

$$
\begin{aligned}
& e^{2 \mu_{2} t}\left\|v_{\xi}(t)\right\|_{L_{w_{2}}^{2}}^{2}+C_{4} \int_{0}^{t} e^{2 \mu_{2} s}\left\|v_{\xi}(s)\right\|_{L_{w_{2}}^{2}}^{2} \mathrm{~d} s \\
& \leqslant C_{1}\left(\left\|v_{0 \xi}(0)\right\|_{L_{w_{2}}^{2}}^{2}+\int_{-\tau}^{0}\left\|v_{0 \xi}(s)\right\|_{L_{w_{2}}^{2}}^{2} \mathrm{~d} s\right) \\
& \quad-b \int_{0}^{t} \int_{\mathbb{R}}\left[\varphi_{\xi}(\xi-c \tau) v(\xi, s)+\varphi_{\xi}(\xi) v(\xi-c \tau, s-\tau)\right] e^{2 \mu_{2} s} v_{\xi}(\xi, s) w_{2}(\xi) \mathrm{d} \xi \mathrm{d} s .
\end{aligned}
$$

Заметим, что вывод оценки (3.20) имеет небольшое отличие от вывода оценки (3.18): надо заменить $1-\varphi(\zeta)$ на $1-u(\zeta, t)-\varphi(\zeta)$, где $\zeta=\xi$ или $\zeta=\xi+c \tau$ при получении оценки (3.10). Эта замена не повлияет на результат. Действительно, из (3.4) вытекает, что $0 \leqslant u^{+}(x, t) \leqslant u_{+}<1$. Поскольку $1-u(\xi, t)-\varphi(\xi)=1-u^{+}(x, t)$, имеем $1-u-\varphi>0$. Подставляя (3.16) с этой модификацией в аналог неравенства (3.14), мы можем не учитывать слагаемое $-u$.

Последнее слагаемое в правой части неравенства (3.20) можно оценить так же, как в работе [7]. Замечая, что $\sup _{\xi \in \mathbb{R}}\left|\varphi_{\xi}(\xi)\right| \leqslant C_{5}, \frac{w(\xi+c \tau)}{w(\xi)} \leqslant 1$, и пользуясь неравенством Юнга $x y \leqslant \varepsilon x^{2}+C(\varepsilon) y^{2}$, имеем

$$
\begin{aligned}
& \left|b \int_{0}^{t} \int_{\mathbb{R}}\left[\varphi_{\xi}(\xi-c \tau) v(\xi, s)+\varphi_{\xi}(\xi) v(\xi-c \tau, s-\tau)\right] e^{2 \mu_{2} s} v_{\xi}(\xi, s) w_{2}(\xi) \mathrm{d} \xi \mathrm{d} s\right| \\
& \leqslant 2 b \varepsilon C_{4} \int_{0}^{t} e^{2 \mu_{2} s}\left\|v_{\xi}(s)\right\|_{L_{w_{2}}^{2}}^{2} \mathrm{~d} s \\
& \quad+2 b C_{5} C(\varepsilon) \int_{0}^{t} e^{2 \mu_{2} s}\|v(s)\|_{L_{w_{2}}^{2}}^{2} \mathrm{~d} s+b C_{5} C(\varepsilon) \int_{-\tau}^{0} e^{2 \mu_{2} s}\left\|v_{0}(s)\right\|_{L_{w_{2}}^{2}}^{2} \mathrm{~d} s
\end{aligned}
$$

Выберем $\varepsilon$ настолько малым, что $2 b \varepsilon C_{5} \leqslant C_{4} / 2$. Из (3.18) вытекает, что

$$
\int_{0}^{t} e^{2 \mu_{2} s}\|u(s)\|_{L_{w_{2}}^{2}}^{2} \mathrm{~d} s \leqslant C\left(\left\|v_{0}(0)\right\|_{L_{w_{2}}^{2}}^{2}+\int_{-\tau}^{0}\left\|v_{0}(s)\right\|_{L_{w_{2}}^{2}}^{2} \mathrm{~d} s\right) .
$$

Подставляя (3.21), (3.22) в (3.20), получаем

$e^{2 \mu_{2} t}\left\|v_{\xi}(t)\right\|_{L_{w_{2}}^{2}}^{2}+\frac{C_{4}}{2} \int_{0}^{t} e^{2 \mu_{2} s}\left\|v_{\xi}(s)\right\|_{L_{w_{2}}^{2}}^{2} \mathrm{~d} s \leqslant C_{6}\left(\left\|v_{0}(0)\right\|_{H_{w_{2}}^{1}}^{2}+\int_{-\tau}^{0}\left\|v_{0}(s)\right\|_{H_{w_{2}}^{1}}^{2} \mathrm{~d} s\right)$.

Не учитывая в этом неравенстве положительное слагаемое $\int_{0}^{t} e^{2 \mu s}\left\|v_{\xi}(s)\right\|_{L_{w_{2}}^{2}}^{2} \mathrm{~d} s$, имеем

$$
e^{2 \mu_{2} t}\left\|v_{\xi}(t)\right\|_{L_{w_{2}}^{2}}^{2} \leqslant C_{6}\left(\left\|v_{0}(0)\right\|_{H_{w_{2}}^{1}}^{2}+\int_{-\tau}^{0}\left\|v_{0}(s)\right\|_{H_{w_{2}}^{1}}^{2} \mathrm{~d} s\right) .
$$

Лемма 3.5 доказана.

С помощью оценки леммы 3.5 можно доказать следующий результат о сходимости. 
Лемма 3.8. Имеем

$$
\|v(t)\|_{L^{\infty}(-\infty, 0]} \leqslant C_{7} e^{-\mu_{1} t / 3}, \quad t>0 .
$$

ДоказАТЕЛьство. Пусть $I=(-\infty, 0]$. Тогда

$$
\begin{gathered}
\|v(t)\|_{L^{2}(I)}^{2}=\int_{-\infty}^{0}|v(\xi, t)|^{2} \mathrm{~d} \xi \leqslant\|v(t)\|_{L^{\infty}}\|v(t)\|_{L^{1}(I)}, \\
v^{2}(\xi, t)=\int_{-\infty}^{\xi} \partial_{\xi}(v)^{2} \mathrm{~d} \xi=2 \int_{-\infty}^{\xi} v(\xi, t) v_{\xi}(\xi, t) \mathrm{d} \xi
\end{gathered}
$$

откуда по неравенству Гёльдера вытекает неравенство Соболева

$$
\|v(t)\|_{L^{\infty}}^{2} \leqslant 2\|v(t)\|_{L^{2}(I)}\left\|v_{\xi}(t)\right\|_{L^{2}(I)} .
$$

Объединяя (3.23) и (3.24), получаем

$$
\|v(t)\|_{L^{\infty}} \leqslant \sqrt[3]{4}\|v(t)\|_{L^{1}(I)}^{1 / 3}\left\|v_{\xi}(t)\right\|_{L^{2}(I)}^{2 / 3} .
$$

Замечая, что $w_{1}(\xi), w_{2}(\xi) \geqslant 1$ при $\xi \in I=(-\infty, 0]$, мы заключаем из леммы 3.4 и леммы 3.5 , что

$$
\|v(t)\|_{L^{\infty}} \leqslant \sqrt[3]{4}\|v(t)\|_{L^{1}(I)}^{1 / 3}\left\|v_{\xi}(t)\right\|_{L^{2}(I)}^{2 / 3} \leqslant \sqrt[3]{4}\|v(t)\|_{L_{w_{1}}^{1}(I)}^{1 / 3}\left\|v_{\xi}\right\|_{L_{w_{2}}^{2}(I)}^{2 / 3} \leqslant C_{7} e^{-\mu_{1} t / 3} .
$$

Лемма доказана.

Теперь распространим полученную оценку на все пространство $(-\infty,+\infty)$. Следуя идеям из работ [8], [15], установим результат о сходимости при $\xi \rightarrow \infty$.

ЛЕмма 3.9. Имеем

$$
\lim _{\xi \rightarrow \infty}|v(\xi, t)| \leqslant C_{8} e^{-\nu t}, \quad t>0
$$

где $C_{8}>0-$ константа $u \nu=b-a>0$.

ДокАзАтЕЛьство. Из первого уравнения (3.5) вытекает, что

$$
v_{t}+c v_{\xi}-J * v+v+(a+b \varphi(\xi-c \tau)) v \leqslant b(1-\varphi(\xi)) v(\xi-y-c \tau, t-\tau) .
$$

При $\xi \rightarrow \infty$, замечая, что $v_{\xi}(t, \infty)=0$ и $\varphi(\infty)=1-\frac{a}{b}$, получаем

$$
v_{t}(\infty, t)+b v(\infty, t) \leqslant a v(\infty, t-\tau) .
$$

Интегрируя последнее неравенство по $[0, t]$, имеем

$$
v(\infty, t)+b \int_{0}^{t} v(\infty, s) \mathrm{d} s-a \int_{0}^{t} v(\infty, s-\tau) \mathrm{d} s \leqslant v(\infty, 0) .
$$

Легко показать, что

$$
\int_{0}^{t} v(\infty, s-\tau) \mathrm{d} s=\int_{-\tau}^{t-\tau} v(\infty, s) \mathrm{d} s \leqslant \int_{0}^{t} v(\infty, s) \mathrm{d} s+\int_{-\tau}^{0} v_{0}(\infty, s) \mathrm{d} s .
$$


Подставляя (3.26) в (3.25), имеем

$$
v(\infty, t)+(b-a) \int_{0}^{t} v(\infty, s) \mathrm{d} s \leqslant v(\infty, 0)+a \int_{-\tau}^{0} v_{0}(\infty, s) \mathrm{d} s .
$$

С помощью неравенства Гронуолла выводим отсюда, что

$$
v(\infty, t) \leqslant C_{8} e^{-\nu t}
$$

для $\nu=b-a>0$. Лемма доказана.

Положим $\mu=\min \left\{\frac{\mu_{1}}{3}, \nu\right\}$. Из лемм 3.8, 3.9 вытекает

ЛЕмма 3.10. Имеем

$$
\sup _{x \in \mathbb{R}}\left|u^{+}(x, t)-\varphi(x+c t)\right|=\sup _{\xi \in \mathbb{R}}|u(\xi, t)| \leqslant C_{9} e^{-\mu t}, \quad t>0,
$$

где $C_{9}>0$.

Шаг 2. Докажем сходимость $u^{-}(x, t)$ к $\varphi(x+c t)$. Положим $\xi=x+c t$ и определим

$$
v(\xi, t)=\varphi(x+c t)-u^{-}(x, t), \quad v_{0}(\xi, s)=\varphi(x+c s)-u_{0}^{-}(x, s) .
$$

Аналогично шагу 1 получаем следующий результат об устойчивости.

ЛЕмма 3.11. Имеем

$$
\sup _{x \in \mathbb{R}}\left|u^{-}(x, t)-\varphi(x+c t)\right|=\sup _{\xi \in \mathbb{R}}|v(\xi, t)| \leqslant C_{10} e^{-\mu t}, \quad t>0,
$$

әде $C_{10}>0$.

Шаг 3. Наконец, докажем сходимость $u(x, t)$ к $\varphi(x+c t)$. Пользуясь леммами $3.10,3.11$ аналогично доказательству леммы 3.10 из работы [8], мы можем установить сходимость $u(x, t)$ к $\varphi(x+c t)$, а точнее неравенство

$$
\sup _{x \in \mathbb{R}}|u(x, t)-\varphi(x+c t)| \leqslant C_{11} e^{-\mu t}, \quad t>0
$$

для некоторой константы $C_{11}>0$.

Доказательство теоремы 2.1 завершено.

\section{§ 4. Обсуждение результатов}

Мы получили глобальную устойчивость фронтов некритических бегущих волн для уравнения (1.3). Легко видеть, что уравнение (1.3) является частным случаем уравнения (1.1). Чтобы доказать устойчивость фронтов бегущих волн уравнения (1.1) или фронтов критических бегущих волн уравнения (1.3), необходимо дополнительно предположить выполнение условия (1.7). 
Рассмотрим вопрос об устойчивости фронтов бегущих волн для уравнения (1.1). Предположим, что входящая в это уравнение функция $f(u, v)$ удовлетворяет следующим условиям:

(S1) для некоторой фиксированной положительной константы $K$ и фиксированного открытого интервала $I \subset \mathbb{R}$ такого, что $[0, K] \subset I$, имеем $f \in$ $C^{2}(\mathbb{R} \times I, \mathbb{R}), \partial_{2} f(u, v) \geqslant 0$ и $\partial_{i j} f(u, v) \leqslant 0, i, j=1,2$, для $u, v \in[0, K]$, причем

$$
\begin{gathered}
f(0,0)=f(K, K)=0, \quad f(u, u)>0, \quad u \in(0, K), \\
\partial_{1} f(0,0)+\partial_{2} f(0,0)>0>\partial_{1} f(K, K)+\partial_{2} f(K, K) ;
\end{gathered}
$$

$(\mathrm{S} 2)$ функция $\partial_{1} f(u, v)$ ограничена на $\mathbb{R} \times[0, K]$.

Легко видеть, что уравнение (1.1) имеет два постоянных состояния равновесия: $u_{-}=0$ и $u_{+}=K$. Состояние $u_{-}$неустойчиво, а $u_{+}$устойчиво. Фронтом бегущей (со скоростью $c$ ) волны уравнения (1.1), соединяющей постоянные состояния $u_{ \pm}$, называется решение вида $u(x, t)=\varphi(x+c t)$, где функция $\varphi$ монотонна и выполнены равенства

$$
\begin{gathered}
c \varphi^{\prime}(\xi)-(J * \varphi)(\xi)+\varphi(\xi)=f(\varphi(\xi), \varphi(\xi-c \tau)), \\
\varphi( \pm \infty)=u_{ \pm},
\end{gathered}
$$

в которых $\xi=x+c t$ и штрих обозначает $\frac{\mathrm{d}}{\mathrm{d} \xi}$. Существование фронтов бегущих волн уравнения (1.1) может быть доказано с помощью метода суб- и суперрешений и теоремы о неподвижной точке. Для $f(u, v)=v(1-u)$ это было сделано в работе [6].

Положим

$$
\Delta_{c}(\lambda)=J * e^{\lambda \cdot}-1-c \lambda+\partial_{1} f(0,0)+\partial_{2} f(0,0) e^{-\lambda c \tau}
$$

Аналогично [6, лемма 4.3] имеем следующие свойства характеристической функции $\Delta_{c}(\lambda)$.

ПРеДЛОЖЕНИЕ 4.1. Для некоторой константы $c^{*}>0$ выполнено следуюmee:

(a) при $0<c<c^{*}$ функчия $\Delta_{c}(\lambda)$ положительна на $\mathbb{R}_{+}$;

(b) при $c=c^{*}$ бункция $\Delta_{c}(\lambda)$ имеет ровно один нуль $\lambda^{*}:=\lambda\left(c^{*}\right) \in\left(0, c^{*}\right)$ на $\mathbb{R}$, причем второго порядка;

(c) при с > $c^{*}$ функиия $\Delta_{c}(\lambda)$ имеет ровно два вещественных нуля: $\lambda_{1}(c)$ и $\lambda_{2}(c)$, при этом $0<\lambda_{1}(c)<\lambda\left(c^{*}\right)<\lambda_{2}(c)$, бункция $\lambda_{1}(c)$ убываюшая по с и $\lambda_{1}(c) \rightarrow 0$ nрu $c \rightarrow \infty$.

Если уравнение (4.1) допускает пару, состоящую из субрешения и суперрешения, то согласно [6, теорема 3.2] это уравнение имеет монотонное решение, являющееся фронтом бегущей волны уравнения (1.1). Положим

$$
\bar{\varphi}(\xi)=K \min \left\{1, e^{\lambda_{1}(c) \xi}\right\}, \quad \underline{\varphi}(\xi)=\max \left\{0, e^{\lambda_{1}(c) \xi}-q e^{\kappa \lambda_{1}(c) \xi}\right\},
$$

где $q>1$ достаточно велико, $\kappa \in\left(1, \min \left\{2, \lambda_{2}(c) / \lambda_{1}(c)\right\}\right)$, а функции $\lambda_{1}(c)$ и $\lambda_{2}(c)$ определены в предложении 4.1. Легко видеть, что $\bar{\varphi}(\xi)$ и $\underline{\varphi}(\xi)$ образуют 
пару из суперрешения и субрешения уравнения (4.1). Поэтому уравнение (1.1) обладает фронтом бегущей волны.

Определим весовую функцию. Пусть $\xi_{0}$ настолько велико, что

$$
\partial_{1} f\left(\varphi\left(\xi_{0}\right), \varphi\left(\xi_{0}-c \tau\right)\right)+\partial_{2} f\left(\varphi\left(\xi_{0}\right), \varphi\left(\xi_{0}-c \tau\right)\right)<0
$$

где $\partial_{i} f:=\partial_{i} f(1,1), i=1,2$. Для таких $c \geqslant c^{*}$ и $\xi_{0}$ определим весовую функцию следующим образом:

$$
\omega(\xi)=\left\{\begin{array}{lll}
e^{-\lambda^{*}\left(\xi-\xi_{0}\right)}, & \text { если } & \xi \leqslant \xi_{0} \\
1, & \text { если } & \xi>\xi_{0}
\end{array}\right.
$$

Теорема 4.1. Пусть выполнены условия (S1), (S2) и (1.7). Тогда для любого фронта $\varphi(x+c t)$ бегущей со скоростью $c \geqslant c^{*}$ волны уравнения (1.1) с условием $\varphi( \pm \infty)=u_{ \pm}$если начальные данные удовлетворяют условиям

$$
u_{-} \leqslant u_{0}(x, s) \leqslant u_{+} \quad \text { npu } \quad(x, s) \in \mathbb{R} \times[-\tau, 0],
$$

$u_{0}(x, s)-\varphi(x+c s) \in C\left([-\tau, 0], L_{\omega}^{1}(\mathbb{R}) \cap H_{\omega}^{1}(\mathbb{R})\right) u \partial_{s}\left(u_{0}(x, s)-\varphi(x+c s)\right) \in$ $L^{1}\left([-\tau, 0], L_{\omega}^{1}(\mathbb{R}) \cap H_{\omega}^{1}(\mathbb{R})\right)$, то решение задачи $(1.1),(1.2)$ существует, является единственным и таково, что

$$
\begin{gathered}
u_{-} \leqslant u(x, t) \leqslant u_{+}, \quad(x, t) \in \mathbb{R} \times \mathbb{R}_{+}, \\
u(x, t)-\varphi(x+c t) \in C\left([0,+\infty) ; L_{w}^{1}(\mathbb{R}) \cap H_{w}^{1}(\mathbb{R})\right),
\end{gathered}
$$

где функиия $\omega(x)$ определена в (4.2). При этом решение $u(x, t)$ сходится $\kappa$ фронту бегущей волны $\varphi(x+c t)$ экспоненциально по времени для $c>c^{*}$ :

$$
\sup _{x \in \mathbb{R}}|u(x, t)-\varphi(x+c t)| \leqslant C e^{-\nu_{\tau} t}, \quad t>0,
$$

где $C>0 u$

$$
\begin{aligned}
0<\nu_{\tau}<\min \left\{-\partial_{1} f(K, K)-\right. & \partial_{2} f(K, K), \\
& \left.\varepsilon_{1}\left(c \lambda-J * e^{\lambda \cdot}+1-\partial_{1} f(0,0)-\partial_{2} f(0,0) e^{-\lambda c \tau}\right)\right\},
\end{aligned}
$$

$a \varepsilon_{1}=\varepsilon_{1}(\tau)$ таково, что $0<\varepsilon_{1}<1$ для $\tau>0 u \varepsilon_{1}(\tau) \rightarrow 0_{+}$при $\tau \rightarrow \infty$.

Если $c=c^{*}$, то решение $и(x, t)$ сходится $к$ фронту бегущей волны $\varphi(x+c t)$ алгебраически:

$$
\sup _{x \in \mathbb{R}}|u(x, t)-\varphi(x+c t)| \leqslant C(1+t)^{-1 / \alpha}, \quad t>0 .
$$

Действительно, из условий (S1), (S2) легко видеть, что для уравнения (1.1) имеет место принцип сравнения. Теперь можно доказать теорему 4.1 с помощью [23, предложение 3.3] и принципа сравнения, но мы не будем входить здесь в детали. Заметим, что теорема 4.1 справедлива и при $\tau=0$. 
Авторы благодарны рецензентам за их ценные предложения и комментарии по первоначальной версии статьи.

\section{Список литературы}

1. P. W. Bates, P. C. Fife, X. Ren, X. Wang, "Traveling waves in a convolution model for phase transitions", Arch. Rational Mech. Anal., 138:2 (1997), 105-136.

2. J. Carr, A. Chmaj, "Uniqueness of travelling waves for nonlocal monostable equations", Proc. Amer. Math. Soc., 132:8 (2004), 2433-2439.

3. X. Chen, "Existence, uniqueness, and asymptotic stability of traveling waves in nonlocal evolution equations", Adv. Differential Equations, 2:1 (1997), 125-160.

4. P. W. Bates, F. Chen, "Spectral analysis and multidimensional stability of traveling waves for nonlocal Allen-Cahn equation", J. Math. Anal. Appl., 273:1 (2002), 45-57.

5. P.W. Bates, F. Chen, "Spectral analysis of traveling waves for nonlocal evolution equations", SIAM J. Math. Anal., 38:1 (2006), 116-126.

6. S.X. Pan, W.-T. Li, G. Lin, "Travelling wave fronts in nonlocal delayed reaction-diffusion systems and applications", Z. Angew. Math. Phys., 60:3 (2009), 377-392.

7. G. Lv, M. Wang, "Nonlinear stability of traveling wave fronts for nonlocal delayed reaction-diffusion equations", J. Math. Anal. Appl., 385:2 (2012), 1094-1106.

8. M. Mei, C.-K. Lin, C.-T. Lin, J.W.-H. So, "Traveling wavefronts for time-delayed reaction-diffusion equation. I. Local nonlinearity", J. Differential Equations, 247:2 (2009), 495-510.

9. D. H. Sattinger, "On the stability of waves of nonlinear parabolic systems", Advances in Math., 22:3 (1976), 312-355.

10. H. L. Smith, X.-Q. Zhao, "Global asymptotic stability of traveling waves in delayed reaction-diffusion equations", SIAM J. Math. Anal., 31:3 (2000), 514-534.

11. X. Chen, J.-S. Guo, "Existence and asymptotic stability of traveling waves of discrete quasilinear monostable equations", J. Differential Equations, 184:2 (2002), 549-569.

12. Z.-C. Wang, W.-T. Li, S. Ruan, "Travelling wave fronts in reaction-diffusion systems with spatio-temporal delays", J. Differential Equations, 222:1 (2006), 185-232.

13. Z.-C. Wang, W.-T. Li, S. Ruan, "Traveling fronts in monostable equations with nonlocal delayed effects", J. Dynam. Differential Equations, 20:3 (2008), 573-607.

14. M. Mei, C. Ou, X. Q. Zhao, "Global stability of monostable traveling waves for nonlocal time-delayed reaction-diffusion equations", SIAM J. Math. Anal., 42:6 (2010), $2762-2790$

15. M. Mei, C.-K. Lin, C.-T. Lin, J.W.-H. So, "Traveling wavefronts for time-delayed reaction-diffusion equation. II. Nonlocal nonlinearity", J. Differential Equations, 247:2 (2009), 511-529.

16. M. Mei, J. W.-H. So, "Stability of strong travelling waves for a non-local time-delayed reaction-diffusion equation", Proc. Roy. Soc. Edinburgh Sect. A, 138:3 (2008), $551-568$.

17. M. Mei, J. W.-H. So, M. Y. Li, S. S. P. Shen, "Asymptotic stability of travelling waves for Nicholson's blowflies equation with diffusion", Proc. Roy. Soc. Edinburgh Sect. A, 134:3 (2004), 579-594.

18. G. Lv, M. Wang, "Nonlinear stability of travelling wave fronts for delayed reaction diffusion equations", Nonlinearity, 23:4 (2010), 845-873.

19. F. Andreu, J. M. Mazon, J. D. Rossi, J. Toledo, "The Neumann problem for nonlocal nonlinear diffusion equations", J. Evol. Equ., 8:1 (2008), 189-215.

20. C. Cortazar, M. Elgueta, J.D. Rossi, N. Wolanski, "Boundary fluxes for nonlocal diffusion", J. Differential Equations, 234:2 (2007), 360-390. 
21. S. A. Gourley, J. Wu, "Delayed non-local diffusive systems in biological invasion and disease spread", Nonlinear dynamics and evolution equations, Fields Inst. Commun., 48, Amer. Math. Soc., Providence, RI, 2006, 137-200.

22. P. Fife, "Some nonclassical trends in parabolic and parabolic-like evolutions", Trends in nonlinear analysis, Springer-Verlag, Berlin, 2003, 153-191.

23. J.D. Murray, Mathematical biology, Biomathematics, 9, Springer-Verlag, BerlinHeidelberg-New York, 1993.

24. R. Huang, M. Mei, Y. Wang, "Planar traveling waves for nonlocal dispersion equation with monostable nonlinearity", Discrete Contin. Dyn. Syst., 32:10 (2012), 3621-3649.

25. E. Chasseigne, M. Chaves, J. D. Rossi, "Asymptotic behavior for nonlocal diffusion equations", J. Math. Pures Appl. (9), 86:3 (2006), 271-291.

СЯО-ХУАНЬ ВАН

Поступило в редакцию

(XiaOhuAn Wang)

School of Mathematics and Information Science,

20.01 .2013

Henan University, China

Гуань-гын Лв

(Guangying Lv)

School of Mathematics and Information Science,

Henan University, China

E-mail: gylvmaths@126.com

Перевод с английского А. В. Домрина 